\title{
The JET Alfvén Eigenmode Local Manager for the real-time detection and tracking of a frequency-degenerate spectrum of MHD instabilities
}

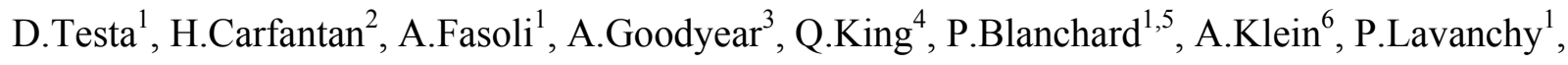 \\ T.Panis ${ }^{1}$, and JET-EFDA contributors* \\ JET-EFDA, Culham Science Centre, OX14 3DB, Abingdon, UK \\ e-mail address of main author: duccio.testa@epfl.ch
}

[1] Ecole Polytechnique Fédérale de Lausanne (EPFL), Centre de Recherches en Physique des Plasmas, Association EURATOM - Confédération Suisse, CH-1015 Lausanne, Switzerland

[2] Université de Toulouse - CNRS, UPS-OMP; IRAP; Toulouse, FR

[3] Culham Centre for Fusion Energy, Culham Science Centre, Abingdon, UK

[4] formerly: JET Joint Undertaking - Joint European Torus, Culham Science Centre, Abingdon, UK

[5] JET-EFDA Close Support Unit, Culham Science Centre, Abingdon, UK

[6] formerly: Plasma Science and Fusion Center, Massachusetts Institute of Technology, Boston, USA

\begin{abstract}
We present the real-time VME system used to detect and track MHD instabilities, and particularly Alfvén Eigenmodes, on the JET tokamak [J.Wesson, Tokamaks, $3^{\text {rd }}$ edition, (Oxford Science Publication, Oxford, 2003), p.617]. This system runs on a $1 \mathrm{kHz}$ clock cycle, and allows performing a real-time, unsupervised and blind detection, decomposition and tracking of the individual components in a frequency-degenerate, multi-harmonic spectrum, using a small number of input data which are unevenly sampled in the spatial domain. This makes it possible to follow in real-time the detected modes as the plasma background evolves, and measure in real-time their frequency, damping rate, toroidal mode-number and relative amplitude. The successful implementation of this system opens a clear path towards developing real-time control tools for electro-magnetic instabilities in future fusion devices aimed at achieving a net energy gain, such as ITER [J.Wesson, Tokamaks, $3^{\text {rd }}$ edition, (Oxford Science Publication, Oxford, 2003), p.711].
\end{abstract}

PACS Classification scheme: 07.05.Dz, 07.05.Kf, 52.35.Bj, 52.55.Fa, 52.70.Ds.

\footnotetext{
* Appendix of F.Romanelli, “Overview of JET Results”, 22 ${ }^{\text {nd }}$ IAEA Fusion Energy Conference, Geneva, 2008; Nucl. Fus. 49 (2009), 104006; weblink: http://iopscience.iop.org/0029-5515/49/10/104006.
} 


\section{1) Introduction.}

The problem of blind and unsupervised real-time detection of the different components in a multiharmonics spectrum using a small number of input data which are un-evenly sampled in the spatial domain is now becoming one of the main aspects required for machine protection and the control of plasma discharges in thermonuclear fusion experiments, and a wealth of literature is available on this subject (for some examples see Chapter3 and Chapter7 and references therein in Ref.[1] and Chapter2 and references therein in Ref.[2]). The method routinely used for this analysis involves sampling of a (relatively) small set of magnetic and non-magnetic signals, often containing some spatial periodicities so as to enhance or eliminate detection of certain components when the input signals are processed appropriately. A real-time algorithm then runs, and a global alarm is generated which may trigger a feedback control mechanism under certain specified conditions. The main drawback of this method is that it can only detect modes when they have become unstable, i.e. when they may have already had some, possibly detrimental, effect on the plasma background parameters.

Conversely, an innovative method has been employed for quite some time now on the JET tokamak [3], which combines active excitation of magnetic field perturbations with a very small amplitude at the plasma edge (maximum intensity $\max \left(\left|\delta \mathrm{B}_{\text {DRIVEN }}\right|\right) \sim 0.1 \mathrm{G}$, i.e. $10^{5}$ times smaller than the typical value of the toroidal magnetic field in JET, $\left.\mathrm{B}_{\mathrm{TOR}} \sim(1-3) \mathrm{T}\right)$ with synchronous real-time detection of the driven perturbations. This is the so-called Alfvén Eigenmodes (AEs) Active Diagnostic (AEAD) system [4, 5], of which the real-time Alfvén Eigenmodes Local Manager (AELM) constitutes one essential, moreover worldwide unique component. This diagnostic system allows the real-time detection and tracking of the driven modes, usually magneto-hydrodynamic (MHD) Eigenmodes supported by the plasma, when still stable, i.e. when the modes have a positive damping $\gamma>0$, and have not yet caused any effect on the plasma background parameters. This is clearly a much more satisfying situation for machine protection and plasma control. The measurements of the mode frequency, damping rate, amplitude and toroidal mode number are passed to the JET Real Time Signal Server (RTSS), and their real-time estimate has been shown to be in very good agreement with the result obtained with a more detailed post-pulse analysis [6-8]. This allows in principle the implementation of a control system and feedback for the modes, so that when the mode's damping rate reduces too much and approaches the marginal stability limit $\gamma / \omega=0$, a control parameter (for instance the edge elongation in the case of AEs [6-9]) can be changed to bring the plasma back to a situation where it is further away from the marginal stability limit. 
A recent upgrade of the AEAD system [10] to allow driving MHD modes with toroidal mode numbers (n) in the range $|n| \leq 30$, has shown conclusively that in this n-number range multiple AEs with different n's can be supported by the plasma at very close-by frequencies, such that the half-width at fullmaximum of two modes (closely related to their damping and growth rate $\gamma / \omega=\operatorname{imag}(\omega) / \omega$ ) is much smaller than their separation in frequency, practically leading to a frequency-degenerate spectrum. This has prompted the development and implementation within the AELM of a new real-time algorithm for the detection, discrimination and tracking of the individual components in such a frequency-degenerate spectrum, which is based on the method of Sparse Representation of Signals [11, 12].

This paper reports on the real-time development of the Sparse Representation method within the AELM hardware and software infrastructure, and on its specific application to the sub-millisecond detection, discrimination and tracking of the individual components in the multi-harmonic, frequencydegenerate spectrum of stable AEs which are excited in the JET tokamak by an array of external antennae used for MHD diagnostic purposes. This paper is organized as follows. In Section2 we briefly review the mathematical foundation of the Sparse Representation method (with a more complete overview given in Appendix-A). Section3 gives an overview of the AEAD system used in JET, with particular attention to its real-time plant control and data analysis hardware and software. Section4 then shows the first examples of the detection and discrimination between the different toroidal components in the multi-harmonic, stable spectrum driven by the AEAD system. Finally, in Section5 we briefly summarize our results and give an outlook towards future work.

\section{2) The Sparse Representation method for the real-time detection and discrimination between the} individual components in an unevenly sampled, frequency-degenerate, multi-harmonic spectrum.

The problem of detection and discrimination between the individual components in a multi-harmonic spectrum which is un-evenly sampled in the spatial domain is common to various fields of physics and engineering [13]. Historically, this problem has been addressed using methods essentially based around the Lomb-Scargle periodograms [14-17], and much work has been done to improve on the limitations of these original methods, essentially in the field of Astronomy and Astrophysics (A\&A). This general measurement problem is further complicated in thermonuclear fusion plasmas, and specifically in large-scale tokamak devices such as JET, by the very low number of measurement points in the spatial domain, due to in-vessel engineering and installation constraints, causing a number of mathematical difficulties. Therefore, analysis method based on the Nyquist criterion cannot in general be used because of aliasing effect. This has prompted the developments and the applications of various methods 
to the analysis of MHD data in thermonuclear fusion plasmas, such as the Singular Value (SVD) [18, 19] and the wavelet [20] decomposition, the Wigner [21], Choi-Williams [22] and Hilbert [23] Transforms, and a generalization of the Lomb-Scargle periodograms [24]. However, none of these methods can be efficiently used for the decomposition of a frequency-degenerate, stable spectrum of MHD modes with the aim to measure their damping rate, because of their mathematical limitations and computational requirements, particularly when real-time, sub-millisecond calculations are needed.

Conversely, a method based on the Sparse Representation of Signals, as implemented in the SparSpec code (freeware available at: http://www.ast.obs-mip.fr/Softwares) has been demonstrated to perform efficiently and correctly the task of a post-pulse [25] and real-time [26] blind and unsupervised signal decomposition of unevenly sampled data using a very small number of measurement points. As some of the mathematical background of this method has been presented elsewhere $[11,12,25,26]$, here we only briefly review its theoretical foundations, with a more compete overview given in Appendix-A to facilitate the reading of this contribution.

In the standard tokamak coordinate system (toroidal angle $\phi$, poloidal angle $\theta$ ), and taking explicitly into account the usual 2D boundary conditions along the longitudinal (toroidal) axis and on the plane perpendicular to it (the poloidal direction), magnetic perturbations can be represented by functions involving toroidal (n) and poloidal (m) harmonics. Considering now the usual case of a perturbation with a specific toroidal mode number $\mathrm{n}$, this can be written as

$$
\psi(\omega, n)=e^{-i \omega t} e^{i n \phi} \sum_{m} A_{m n} e^{i m \theta}
$$

where each mode has one single toroidal mode number, but includes several poloidal harmonics. The aim of toroidal (poloidal) mode numbers detection is to measure the mode numbers $\mathrm{n}(\mathrm{m})$ of the magnetic instabilities present in the plasma and to estimate their amplitude from data acquired with $\mathrm{P}$ detectors unevenly positioned at angles $\phi_{\mathrm{p}}\left(\theta_{\mathrm{p}}\right), \mathrm{p}=\{1, \ldots, \mathrm{P}\}$. Hence each measurement $y_{p} \in \square$ can be mathematically modelled as

$$
y_{p}=\sum_{l} \alpha_{l} \exp \left(\operatorname{in}_{l} \phi_{p}\right)+\varepsilon_{p}
$$

with $l=\{1, \ldots, L\}$, where $n_{1}$ and $\alpha_{1}$ are the unknown mode numbers and complex amplitudes, respectively, $L$ the unknown number of modes and $\varepsilon_{\mathrm{p}}$ corresponds to the noise on the data for the given 
p-th sensor. Note that the data $y_{p}$, the amplitudes $\alpha_{l}$ and noise $\varepsilon_{p}$ are all complex-valued quantities: specifically, $\alpha_{1}$ represents the amplitude and the phase of the modes being measured.

We then solve the problem of determining the unknowns $n_{1}$ and $\alpha_{1}$ using a Sparse Representation method based on minimizing the L1-norm penalized criterion:

$$
J_{0}(x)=\|\mathbf{y}-W \mathbf{x}\|^{2}+\lambda\|\mathbf{x}\|_{1}=\|\mathbf{y}-W \mathbf{x}\|^{2}+\lambda \sum_{k=-K}^{K}\left(\left|x_{k}\right|\right)
$$

Here $\mathbf{y}$ is the vector of data taken at position $\phi_{\mathrm{p}} ; \mathbf{x}$ is the vector of complex $M=2 K+1$ components representing $\mathbf{y}, \mathbf{W}$ is the spectral window (i.e. $\mathbf{W}$ is the matrix of exponential terms $W_{l, p}=\exp \left(\operatorname{in}_{l} \phi_{p}\right)$ ), $\lambda$ is a penalization parameter related to the noise level, and the L1-norm is defined as the sum of the amplitudes of all the non-zero components in the measured spectrum. Many computationally efficient algorithms have been developed to solve eq.(3), and the SparSpec code uses one based on an iterative Block Coordinate Descent $[11,12]$ algorithm. A version of this algorithm has recently been adapted to perform the required mode decomposition analysis using the rather modest computational resources available to process real-time JET data [26].

\section{3) The active MHD diagnostic system in use at JET and the Alfvén Eigenmode Local Manager.}

The MHD spectroscopy is a diagnostic technique that uses waves that are naturally supported by the plasma to measure the parameters that determine the dispersion relation, absorption and propagation, damping and growth of these waves [5, 27]. One example of such waves is Alfvén Eigenmodes (AEs): these modes are particularly important as they are a natural Eigenmode of any magnetically confined plasma, and because the fusion-produced $\alpha$ s are born with a supra-thermal speed that is typically superAlfvénic in the usual thermonuclear tokamak plasma conditions. Therefore, resonant interaction with AEs is the first wave-particle interaction that the as encounter during their thermalization: hence, this mechanism for phase-space and spatial diffusion needs to be controlled appropriately to guarantee good confinement of the $\alpha$ s themselves $[28,29]$. A simple active method to drive and detect low amplitude modes in the plasma was pioneered and used in many different plasma conditions in the JET tokamak [4]. This is the so-called Alfvén Eigenmodes Active Diagnostic (AEAD) system, and fig1 shows a very simplified schematic overview to illustrate its main features. The AEAD system principally consists of: 
1. the AE exciter, built upon a function generator and a (high-power) amplifier connected to a set of in-vessel antennae (up to 8 in our case), whose aim is to send power into the plasma in order to drive a very small magnetic perturbation at the plasma edge, with maximum intensity $\max \left(\left|\delta \mathrm{B}_{\mathrm{DRIVEN}}\right|\right) \sim 0.1 \mathrm{G}$, i.e. $10^{5}$ times smaller than the typical value of the toroidal magnetic field in JET, $\mathrm{B}_{\mathrm{TOR}} \sim(1-3) \mathrm{T}$;

2. a receiver, built upon synchronous detection units, which is collecting signals from a set of invessel detectors for electro-magnetic fluctuations, such as magnetic pick-up coils, electron cyclotron emission and reflectometry measurements; this receiver is also connected to the real-time AE Local Manager (AELM) to allow for the detection and tracking of antenna-driven plasma resonances.

The AE exciter is built upon a $5 \mathrm{~kW}$ class-B power amplifier capable of delivering up to a maximum $\mathrm{I}_{\mathrm{ANT}} \sim 10 \mathrm{~A}$-peak and a maximum $\mathrm{V}_{\mathrm{ANT}} \sim 1 \mathrm{kV}$-peak in the frequency range $10 \mathrm{kHz} \rightarrow 500 \mathrm{kHz}$ to each of the 8 in-vessel antennae. The antennae are installed in two groups of four closely-spaced units located at two toroidally opposite positions but at the same poloidal location [10]. This allows the magnetic fluctuations to be driven with a sufficiently high $\left|\delta \mathrm{B}_{\text {DRIVEN }}\right|>10^{-3} \mathrm{G}$ at the plasma edge for a spectrum of toroidal mode number extending up to $|\mathrm{n}| \sim 30$.

The plasma response to the antenna-driven perturbation is principally measured on a selected subset of signals using synchronous detection [4]. Looking at any electro-magnetic turbulence measurements, such as the signals from the magnetic pick-up coils, we would see that we measure a multitude of mixed frequencies. Conversely, for real-time use of the AEAD diagnostic, we need to measure only the plasma response at the frequency corresponding to the antenna excitation, i.e. the synchronous component. The purpose of the synchronous detection system is to select only the portion of the input signal which is at the same frequency as the one chosen for the excitation of the modes: this also correspondingly reduces the required bandwidth of the data acquisition system (which has a $1.25 \mathrm{kHz}$ sampling rate for $12 \mathrm{sec}$ of each JET pulse, compared to the $1 \mathrm{MHz}$ sampling rate typical for the other JET fluctuation measurements), and also filters-out all other unwanted frequency components. The measured signal is then directly in relation (i.e. it is synchronous) to the launched antenna signal. The synchronous detection hardware works conceptually by applying a mixer with the synchronous inphase (I) and quadrature (Q) components to the incoming differential signal, and then applying a lowpass filter with a $<100 \mathrm{~Hz}$ bandwidth to generate the output [cosine (I), sine (Q)] DC components. A schematic diagram of the synchronous detection hardware is shown in fig2.

Analogue synchronous detection is essential for the real-time applications (with plant protection and control using 24 channels, data analysis using 8 channels) to reduce the bandwidth required for data acquisition (a total of 48 channels in our case) and avoid the need for computationally expensive FFT- 
type algorithms. Post-pulse analysis can obviously still be performed using the much larger set of raw signals (between 32 and 80 additional channels depending on the operational setup) having the required sampling rate (at least $1 \mathrm{MHz}$ ) via software-based synchronous detection. Further technical details on the active MHD diagnostic system used at JET can be found in $[4,7,8,10]$ and the references therein. One essential and worldwide unique component of the AEAD system is the AELM. The AELM is a digital VME plant control system, used to monitor and control the operation of the AEAD plant in real time, with a $1 \mathrm{kHz}$ clock-rate. A schematic overview diagram for the AELM is shown in fig3.

The AELM receives the feedback inputs for the AEAD system via ADC modules called VAJ1. These input data consist of 8 magnetic pick-up coils used for mode detection, 22 engineering signals, used for plant control and normalization of the driven excitation spectrum, and two frequency deviation signals from the Ion Cyclotron Resonance Frequency (ICRF) antennae, used for RF beatwave control operation [30]. The AELM is supplied with the main plasma parameters via the RTSS, such as the plasma density, the toroidal magnetic field and the plasma current; these signals are required to compute in real-time the AE frequency. The AELM is also supplied with the overall JET timing signals originating from the Composite Time and Trigger Signal (CTTS) system.

In addition to providing the timing control sequence for the AEAD system, the AELM produces a number of outputs sent back to the AEAD plant through DAC modules, again using the VAJ1 units. The main AELM output is the AE frequency, called $\mathrm{F}_{\mathrm{REF}}$, which is a modulated DC voltage connected to the Voltage Controlled Oscillator (VCO) input of a function generator: the VCO frequency output is feedback controlled so that tracking can occur.

\section{The AELM crate contains four main components:}

1. a VME Crate Service Module, acting as the VME system controller;

2. a Real-Time Processor (RtProc), operating on a $1 \mathrm{kHz}$ cycle: this card is only activated during a JET pulse, with the main purpose of performing mode detection, discrimination and tracking using one of two user-selectable options, the "SimpleSum" and the "SparSpec" algorithms;

3. a Communications Processor (CommsProc), used to setup pre-pulse information, to synchronize the RtProc with important time points within the pulse and finally to communicate data recorded during the pulse for archiving;

4. four off-the-shelf Pentland Systems MPV956 analogue and digital input/output cards (VAJ1).

The selection of the run-time parameters for the operation of the AEAD system is performed via a configuration panel, and fig4 shows its main operating window for the SparSpec algorithm when the real-time value of the frequency of Toroidal Alfvén Eigenmodes (TAEs) is used for frequency control. 
There are two possibilities for selecting the frequency source $\mathrm{F}_{\mathrm{REF}}$ : a pre-defined waveform and an algorithm to evaluate the real-time AE frequency, based on the measurement of the magnetic field, plasma current and density (the usual selection, labelled "AELM"). This choice is made by selecting the "Fref Source" tab on the AELM configuration panel (see fig4). If the "AELM" mode is selected for the $\mathrm{F}_{\mathrm{REF}}$ source, then, as shown in fig4, a number of additional parameters need to be set according to the formula:

$\mathrm{F}_{\mathrm{REF}}[\mathrm{kHz}]=32.7 \times\left(\mathrm{F}_{\mathrm{REF}}\right.$ Multiplier $\left.\times \operatorname{Itf}[\mathrm{A}]\right) /\left(\operatorname{sqrt}\left(\right.\right.$ MassNumber $\times$ Density $\left.\left.\left[10^{18} \mathrm{~m}^{-2}\right]\right)\right) \times$ (Ip[A] / IpNorm[A]).

In eq.(4), Itf is the current in the toroidal field coils, Ip is the toroidal plasma current (IpNorm being a normalization factor), Density is the line-averaged plasma density, MassNumber accounts for the main plasma gas and $\mathrm{F}_{\mathrm{REF}}$ Multiplier is a multiplicative scaling factor. The maximum operating frequency for the AEAD plant (" $F_{M A X}$ ") is set by the corresponding tab $\left(F_{M A X}=500 \mathrm{kHz}\right.$ in the case shown in fig4).

Once the choice of the frequency source has been made, the scanning or tracking mode may be selected. If frequency scanning is disabled, the AELM will output exactly the frequency set using the $F_{R E F}$, i.e. the antenna frequency $F_{A N T}$ is in the range $0 \leq F_{A N T}=F_{R E F} \leq F_{M A X}$. When the scanning mode is enabled, a pre-defined up/down symmetric (repetitive) sweep, with parameters setup via the AELM, is added to the chosen $\mathrm{F}_{\mathrm{REF}}$. The values for the scan half-width ("ScanWidth") and speed ("ScanSpeed") can be selected separately for the limiter and X-point ("diverter") magnetic configurations. When the tracking mode is enabled (which can only be used if scanning is enabled), the real-time mode detection algorithm is used to lock the AELM frequency scan around the frequency of the detected modes, so as to track their evolution in real-time. This is done by reversing the direction of the frequency sweep when certain resonance conditions are met (see below for further details), or at the extrema of the frequency scan $\mathrm{F}_{\mathrm{REF}} \pm$ ScanWidth when these conditions are not met.

To detect and track in real-time the individual resonances corresponding to antenna-driven, stable plasma modes, the AELM linearly sweeps the antenna frequency around an initial guess for the mode's resonance. One of two algorithms can be selected to derive a single amplitude and phase pair that will be used for mode detection and tracking: the original ( $\sim 10$ years old) "SimpleSum" algorithm combines up to 8 real and imaginary pairs from the total 8 input data available to obtain one single pair of real and imaginary components, whereas the newly developed "SparSpec" algorithm can also accept up to 8 input real and imaginary pairs but produces many output amplitude and phase pairs. When using the SparSpec tracking mode, the AELM has two methods, "highest" and "any", for selecting the pair to use 
for tracking: the highest method picks the pair with the greatest amplitude, whereas the any method looks for a pair where the amplitude is above a given threshold; if a resonance is detected, this pair will continue to be selected until tracking is lost when the search for a new pair will commence. When the AE resonance is met, the exciter frequency is swept back and forth around it. A Lorentzian model fit of the antenna/plasma transfer function is used to obtain the mode frequency and quality factor in realtime, and the value of the magnetic perturbation at the different probes. This simple real time estimate of the mode frequency and damping rate from the centre and width of the driven resonance follows very closely the value obtained from a post-pulse analysis [6-8].

Figure5 shows an illustrative example of the AEAD plant in the tracking mode of operation. In the fullfrequency spectrum (shown in the left frame) we see a triangular waveform that sometimes becomes very narrow in an otherwise completely clean portion of the fluctuation spectrum: this is the antenna frequency, which was set to look for resonances around the frequency of an $n=3$ TAE as evaluated in real-time for that shot (i.e. around $200 \mathrm{kHz}$ ). In the right frame, we have the synchronous detected signal $\left(\left|\delta B_{M E A S}\right|\right)$ from one magnetic pick-up coil, showing the real-time TAE frequency $\left(f_{\mathrm{TAE}}\right)$ and the antenna frequency. Narrow sweeps of the antenna frequency occur when the complex-valued $\delta B_{\text {MEAS }}$ (shown in the two inserts) is sufficiently close to the pre-set resonant shape, i.e. it describes a circle in the complex plan representation with a corresponding bell-shape in the $\left|\delta B_{\text {MEAS }}(\omega)\right|$ representation (Remember that $\delta \mathrm{B}_{\text {MEAS }}$ is a complex-valued quantity as it is the synchronously acquired measurement, i.e. it has both the in-phase (I) and the quadrature (Q) components). We are then tracking the mode, and its frequency $\left(\mathrm{f}_{\mathrm{MEAS}}\right)$ and damping $\left(\gamma_{\text {MEAS }}\right)$ are measured in real-time. Such narrow sweeps occur between $\mathrm{t}=8 \rightarrow 10 \mathrm{sec}$ and $\mathrm{t}=11 \rightarrow 14 \mathrm{sec}$. The much larger frequency sweeps indicate that no resonance close to the pre-set value has been detected in real-time: we are then scanning for a mode, and the AELM looks for antenna-driven plasma resonances in a different frequency range. This approach allows the detection and tracking of hundreds of individual resonances during one single tokamak discharge, which are guaranteed to be the same mode, i.e. to have the same n-number, if the SparSpec algorithm has been used.

As shown in fig4, the AELM configuration panel has many tabs requiring user input for the mode detection algorithm, which is selected via the tab "DetectorSignal". Figure6 shows graphically an intuitive description of the use of some of these parameters. At the beginning of each frequency sweep, if a mode has been detected (via the SimpleSum or both the SparSpec methods), we check that the amplitude of the signal detected at the magnetic probes $\left(\omega\left|\delta \mathrm{B}_{\mathrm{MEAS}}\right|\right)$ and its speed $\left(\left|\delta \mathrm{B}_{\mathrm{MEAS}} / \mathrm{I}_{\mathrm{ANT}}\right|\right)$ are above the set thresholds (bottom left plot: AmpThresh $=0.65 \times 10^{-9}[\mathrm{~T} / \mathrm{s}]$ and SpeedThreshStart $=5 \times 10^{-}$ 
$\left.{ }^{13}[\mathrm{~T} / \mathrm{A}]\right)$. If these conditions are satisfied, we start computing the time-integrated twist (i.e. the angle $\operatorname{imag}\left(\delta \mathrm{B}_{\mathrm{MEAS}}\right) / \mathrm{real}\left(\delta \mathrm{B}_{\mathrm{MEAS}}\right)$, see the bottom right plot) using the complex plane representation of $\delta B_{\text {MEAS }}(\omega)$ (top plot). We estimate that the antenna-driven plasma resonance has been fully identified when the integrated twist value exceeds the set threshold $=2 \mathrm{rad}$ and if the speed at the supposed end of the frequency sweep is below the set threshold SpeedThreshEnd $=6 \times 10^{-13}[\mathrm{~T} / \mathrm{A}]$. Hence, at this moment the direction of the frequency sweep is reversed (as shown in the insert in the top plot) in an attempt to follow the evolution of the same mode as the background plasma evolves.

\section{4) Real-time, blind, unsupervised detection and discrimination of different toroidal components in a frequency-degenerated spectrum of MHD instabilities using the AELM.}

One of the first results obtained with the AEAD diagnostic system in the most recent JET experimental campaigns has been that, despite the very low magnetic field driven by the antennae for medium-n AEs (in the plasma core we have $\left|\delta \mathrm{B}_{\text {DRIVEN }}\right| \sim 1 \times 10^{-3} \mathrm{G}$ for $\mathrm{n}=5$ compared to $\left|\delta \mathrm{B}_{\text {DRIVEN }}\right| \sim 5 \times 10^{-2} \mathrm{G}$ for the $\mathrm{n}=1$ and $n=2$ modes), many modes with $|n| \sim 0-12$ and very low-damping rate $\gamma / \omega<0.2 \%$ were found to be simultaneously excited in plasmas without populations of resonant fast ions [7,8]. Correct real-time detection and n-number discrimination of these modes is particularly important as their low intrinsic (i.e. without any fast ion drive) damping rate makes them very prone to become unstable if resonant fast ions were present in the plasma. It is specifically this experimental observation that has prompted the development of the more sophisticated real-time algorithm for mode-number recognition based on the SparSpec algorithm, which has now almost completely replaced the original SimpleSum method.

The capability to perform a blind and unsupervised real-time detection and tracking of the individual ncomponents in the antenna driven spectrum constitutes an invaluable tool, which is unique to the JET tokamak. It provides accurate testing for the code prediction for the damping rate of Alfvén Eigenmodes (see for instance the results presented in [9]), as it is paramount that the same mode be measured throughout the parameter scan. The full implementation of the SparSpec algorithm in the AELM software now allows a detailed quantitative analysis of the recent measurements made with the AEAD system in JET. Mode numbers can be directly separated in real-time and individually tracked to measure the changes in the mode frequency and $\gamma / \omega$ during the evolution of the plasma background. It is also paramount that the real-time detection matches the results obtained via the more complex and accurate post-pulse analysis method, and this has been discussed and demonstrated in detail in [7,8].

Figure $7 \mathrm{a}$ shows an example of the real-time detection and tracking of individual $n=3, n=5$ and $n=7$ TAEs for the JET shot \#77417, where the excitation system was configured to drive predominantly n- 
odd modes, with $\max \left(\left|\delta B_{\text {DRIVEN }}(n)\right|\right)$ in the range $|n|<5$. For this shot, the AELM tracking algorithm was configured in the "SparSpec-highest" mode, with Amp.Thresh $=3 \times 10^{-9} \mathrm{~T} / \mathrm{s}$, Speed.Thresh $=3 \times 10^{-13} \mathrm{~T} / \mathrm{A}$ (using the same value for the start and end speed thresholds), Minimum.Twist $=2 \mathrm{rad}, \lambda_{\mathrm{NORM}}=0.9$ (the normalization value for the L1-norm criterion, see Appendix-A), $\mathrm{N}_{\mathrm{MAX}}=10$ (the maximum toroidal mode number to be detected) and detection for modes with $n=3 \rightarrow 10$ (i.e. only co-current propagating). The digital signals shown at the bottom of fig7a indicate whether detection and tracking of a certain mode has been successful: if the validity flag is set to high=1 then the corresponding mode has been correctly detected and tracking is occurring, otherwise the digital signal is set to low $=0$ (note that it is in principle possible to have a valid $n=\mathrm{N}_{\text {MAX }}$ mode as long as the validity flag is $=1$ : therefore, it is the validity flag that denotes "good" or "bad" tracking for the $n=\mathrm{N}_{\text {MAX }}$ mode). At the start of each real-time scan, the mode value was initialized to a bad value of $\mathrm{n}=\mathrm{N}_{\mathrm{MAX}}$ and its associated digital validity flag set to 0 (not shown in fig7a, where we only show the digital flags for certain specific n's to reduce cluttering). In fig7a we note that the antenna frequency waveform $\mathrm{F}_{\mathrm{TAE}}$ follows closely the real-time evolution of the reference TAE frequency $F_{\mathrm{REF}}$, and that the $n=3$ mode is the one most detected in realtime. This is consistent with the fact that (a) the excitation spectrum was larger for $|\mathrm{n}|<5$, and that (b) counter-current propagating modes $(\mathrm{n}<0)$ and modes with $\mathrm{n}<3$ were not selected for real-time detection via the AELM. These results are confirmed by a more detailed post-pulse analysis using the full implementation of the SparSpec algorithm, which includes detection and discrimination between all modes up to $|\mathrm{n}|=30$, using $\lambda_{\mathrm{NORM}}=0.7$ to help in resolving also lower-amplitudes modes. This analysis is shown in fig $7 b$, where we plot the mode frequency and damping rate $\gamma / \omega$ as sorted for decreasing mode amplitude for all the detected TAEs, but only few values for the normalized excitation spectrum $\left|\delta \mathrm{B}(\mathrm{n}) / \mathrm{sum}\left(\mathrm{I}_{\mathrm{ANT}}\right)\right|$ to reduce cluttering.

By comparing in detail the results shown in fig7a and $7 \mathrm{~b}$, we note that in real-time we pick-up only a fraction of the entire measurable dataset of toroidal mode numbers as we have a very stringent computational time constraint (all the real-time calculations must be completed within $\sim 850 \mu$ s to stay within a safe limit compatible with the $1 \mathrm{~ms}$ clock-rate of the AELM and RTSS systems), whereas with the post-pulse analysis we can indeed obtain the entire dataset of measurements. We can then ascertain that the post-pulse and the real-time analyses agree within the real-time calculations constraints, i.e. with the post-pulse analysis not only showing the data obtained in real-time, but also those that, effectively, we had to discard in real-time to meet the computational time requirements. It is only by showing such a comparison, i.e. including the full dataset obtained post-pulse, that we believe we can convincingly prove the validity of our approach for the real-time calculations. 
To illustrate in more details the logic of the SparSpec tracking algorithm as implemented in real-time in the AELM, fig8 shows an example of the real-time time twist and speed calculations for the $\mathrm{n}=11 \mathrm{TAE}$ mode in the JET shot $\# 77790$, where the excitation system was configured to drive predominantly nodd modes, with $\max \left(\left|\delta B_{\text {DRIVEN }}(n)\right|\right)$ in the range $|n| \sim 5-10$. For this shot, we used the tracking mode SparSpec-highest, with $\mathrm{N}_{\mathrm{MAX}}=15, \lambda_{\mathrm{NORM}}=0.95$, Amp.Thresh $=1 \times 10^{-9} \mathrm{~T} / \mathrm{s}$, Speed.Thresh $=3 \times 10^{-13} \mathrm{~T} / \mathrm{A}$ (using the same value for the start and end speed thresholds), Minimum.Twist=2.5rad, and detection for modes with $n=[-15,-11,-9,-7,-5,5 \rightarrow 11]$. Comparing in fig8a the status of the digital flags for the amplitude (SigAmp.11) and speed (Speed.11) for the $n=11$ mode, we note that it is only when these two signals are concurrently high that the corresponding digital flag for the differential twist (TwistRate.11) is also set to high, hence the integrated twist is being calculated and tracking of the $\mathrm{n}=11$ mode can occur (mode signal set to $=11$ ) if the integrated twist exceeds the set threshold. Specifically, this mostly happens in the time window $5.0<\operatorname{time}(\mathrm{sec})<9.5$ (an highlight of this is shown in fig8b), and rarely in other time intervals, indicating a subtle dependence of the stability of the $\mathrm{n}=11$ mode as function of the background plasma parameters.

Finally, when combining the real-time mode number, frequency and damping rate data obtained in various shots (hence with different plasma configurations and background parameters) when different setups were used for the SparSpec tracking algorithm within the AELM (such as different values for $\mathrm{N}_{\mathrm{MAX}}$, Amp.Thresh, Speed.Thresh, Minimum.Twist, and the n's selected for real-time detection), and using different excitation spectra, we can compare these data with the corresponding ones obtained with the post-pulse analysis. This allows us to obtain a statistical analysis of the accuracy of the real time measurements. For this, we consider the expectation value on the mode number, frequency and damping rate as the one given by the post-pulse analysis, use the actual post-pulse estimation on the errors on these quantities, which are typically around the values presented in [7] (i.e. $\sim 10 \%$ on the n's and frequency, $\sim 25 \%$ on $\gamma / \omega)$ and define a confidence level for the real-time measurements as:

$$
\text { confidence level }=\exp \left(\frac{(\text { RealTimeData }- \text { PostPulseData })^{2}}{(\text { VariancePostPulseData })^{2}}\right)
$$

The Gaussian confidence level data obtained with this procedure are shown in fig9 as function of the toroidal mode number for all the modes for which we have sufficient statistics. We note that the confidence level is quite high for all the real-time data obtained with the SparSpec tracking algorithm, at least exceeding 0.8 for all mode numbers in the range $-8<\mathrm{n}<12$. This proves that the SparSpec real- 
time mode detection and tracking algorithm can indeed be used as a valuable diagnostic tool for blind and unsupervised mode discrimination in a multi-components and frequency-degenerate spectrum.

\section{5) Summary and Conclusions.}

In this work we have reported on the application of a new method for the real-time and unsupervised detection and decomposition of a multi-harmonic and degenerate spectrum of high-frequency magnetic instabilities measured on the JET tokamak, i.e. one where the frequency separation between the various components is less than their half-width at full-maximum. This method uses real-time measurements performed on a $1 \mathrm{~ms}$ time base, processed by the AELM, which is a VME system interfacing with the JET RTSS system. The real-time mode detection and discrimination algorithm is based on the Sparse Representation of signals, derived from its original applications to astronomical data via the SparSpec code. The development of this method was prompted by the first measurements in the JET tokamak of such a frequency degenerated spectrum of antenna-driven AEs, with toroidal mode numbers $|\mathrm{n}| \sim 3-15$. The real-time (and post-pulse) implementation of the SparSpec algorithm on JET has allowed a complete, accurate and numerically efficient analysis of these measurements, which would have not been possible otherwise. For post-pulse analysis, the antenna-driven spectrum can be decomposed and analyzed for one whole frequency scan typically within (2-3) $\mu$ s of CPU time using the full (more complex and more accurate) implementation of the SparSpec algorithm. With an SVD decomposition algorithm similar to the one presented in [19], requiring a combinatorial exploration of all possible solutions and an a-posteriori thresholding scheme to determine the correct ones, or using wavelet-based schemes, such as those presented in [20], this analysis would require a CPU time usually in excess of $150 \mathrm{~ms}$ for each individual frequency scan. Such computational requirements make it impossible to use these schemes for real-time analysis. Conversely, using the rather modest computational resources allocated to the real-time analysis of the AEAD data within the AELM hardware and software (a $1 \mathrm{GHz}$ PowerPC with a $512 \mathrm{MB}$ RAM running on a $1 \mathrm{kHz}$ clock-rate), the multi-components antenna-driven spectrum can be fully resolved within typically $\sim 650 \mu$ s for each $1 \mathrm{~ms}$ clock-cycle. Finally, the results for the mode frequency, damping rate, mode numbers (and scaled mode amplitude) obtained with the real-time SparSpec algorithm are in good agreement both statistically and on a shot-by-shot basis with those obtained with the post-pulse implementation of this algorithm, confirming that the AELM system can indeed be valuably used for real-time analysis for plasma control purposes.

For JET, and more generally fusion plasmas, further applications of this new method based on the Sparse Representation of Signals open interesting and very useful perspectives for the concurrent real- 
time detection and control of different MHD instabilities, as they can be discriminated very accurately. This allows specifically tailored control schemes to be put in place for each individual mode, hence improving the overall control of the plasma operation and performance. This will be particularly important for future experiments approaching the burning plasma conditions, such as ITER, where realtime control of the stability of the fusion born alphas in the background "sea" of frequency-degenerate MHD modes that are expected to occur in such conditions, represents one of the key ingredients required to achieve a net fusion energy gain. Furthermore, and while specifically applied for the analysis of astronomical data and toroidal mode numbers in thermonuclear fusion plasmas in a tokamak device, the use of Sparse Approximations methods are ideally suited for applications to all domains of data analysis, control engineering and system design where an efficient decomposition of a multiharmonics degenerate spectrum is required from irregularly sampled data. An example of this work is the application of the SparSpec algorithm to the analysis and optimization of the baseline design for the diagnostic system intended for the measurement of high-frequency instabilities in ITER [31].

\section{Acknowledgements.}

This work, supported by the European Communities under the contract of Association between EURATOM and CRPP-EPFL, was carried out within the framework of the European Fusion Development Agreement. This work was also partly supported by the Swiss National Science Foundation. The views and opinions expressed herein do not necessarily reflect those of the European Commission. The Authors would like to thank the various members, past and present, of the CRPP, MIT and JET staff that have contributed to the design, installation, commissioning and operation of the Alfvén Eigenmode Active Diagnostic system over more than 10 years of experimental runs at JET. The Authors would also like to thank the Reviewers for their useful comments and suggestions. 


\section{Appendix-A: Sparse Representations and the SparSpec code.}

In the standard tokamak coordinate system (toroidal angle $\phi$, poloidal angle $\theta$ ), and taking explicitly into account 2D boundary conditions along the longitudinal (toroidal) axis and on the plane perpendicular to it (the poloidal direction), magnetic perturbations can be represented by functions involving toroidal $(\mathrm{n})$ and poloidal $(\mathrm{m})$ harmonics. Considering now the usual case of a perturbation with a specific toroidal mode number $\mathrm{n}$, this can be written as

$$
\psi(\omega, n)=e^{-i \omega t} e^{i n \phi} \sum_{m} A_{m n} e^{i m \theta}
$$

where each mode has one single toroidal mode number, but includes several poloidal Fourier harmonics. The aim of toroidal (poloidal) mode numbers detection is to detect the mode numbers $\mathrm{n}(\mathrm{m})$ of the magnetic instabilities actually present in the plasma and to estimate their amplitude from data acquired with detectors unevenly positioned at angles $\phi_{p}\left(\theta_{p}\right)$ in radians, $p=\{1, \ldots, P\}$ being the suffix labelling the individual sensors used for the measurement. Mathematically, each complex measurement $\mathrm{y}_{\mathrm{p}}$ is then modelled as:

$$
y_{p}=\sum_{l=1}^{L} \alpha_{l} e^{i n_{l} \phi_{p}}+\varepsilon_{p}
$$

where $n_{1}$ and $\alpha_{1}$ are the unknown mode numbers and complex amplitudes, respectively, $L$ the unknown number of modes and $\varepsilon_{\mathrm{p}}$ corresponds to the (complex-valued) noise on the data for the given $\mathrm{p}$-th sensor. This problem, which amounts to fitting multiple cisoids to the input data, is a very general signal processing problem which arises in many fields of physics. However, it is particularly difficult in the case of tokamak plasma physics because of the low number of unevenly sampled data, due to unavoidable installation constraints on the measurement devices. Such a spectral analysis problem from irregularly sampled data is very common in Astronomy and Astrophysics (A\&A), where time series acquisition usually suffer from incomplete temporal coverage, in particular periodic gaps caused by the Earth's rotation and revolution, and a-periodic interruptions due to the weather. Many methods have been proposed in the fields of A\&A to improve the analysis of such irregularly sampled time series, based on generalizations of the Lomb-Scargle periodogram [14, 15] and Date-Compensated Discrete Fourier Transform [32]. These methods involve iterative analysis [17], generally used when dealing with a large number of data points, or fitting periodic signals (Phase Dispersion Minimization [33], 
string length method [34]) to short data strings. Such methods, however, are inadequate when there are several temporal frequencies and too few measurement points.

The mathematical problem described by eq.(A2) can be expressed equivalently in the Fourier spatial domain (i.e. using a Fourier transform with respect to the angular position $\phi$ ) as:

$$
\hat{y}(v)=\sum_{p=1}^{P} y_{p} e^{i v \phi_{p}}=\hat{W}(v) * \sum_{l=1}^{L} \alpha_{l} \delta\left(v-n_{l}\right)+\hat{\varepsilon}(v)
$$

where $\delta$ is the Dirac delta function, the "^" symbol indicates the Fourier transform of the data $(\hat{y}(v))$ and noise $(\hat{\varepsilon}(v))$ samples, the symbol "*" is the convolution operator and $\hat{W}(v)$ is the spectral window of the sampling scheme. It can be shown that the difficulty of the spectral analysis problem is closely related to properties of the spectral window, such as the height of secondary lobes. In A\&A, when accounting for long times series, high secondary lobes are due to periodicities, typically daily, of the temporal gaps in the measurement (see figA1, top frame), while in thermonuclear tokamak plasmas these lobes are due to regularities in the sampling (for instance when using a spacing larger than the Nyquist condition) and to the low number of sensors (see figA1, bottom).

When applied to thermonuclear plasma physics, the problem has some additional specificity. First, the data $y_{p}$ are complex-valued, implying that the Fourier transform of the data does not satisfy the Hermitian property $\hat{y}(-v)=\hat{y}^{*}(v)$ as in the spectral analysis of real-valued data. Second, the modes numbers $\mathrm{n}_{1}$ can only take positive or negative integer values, while in the general spectral analysis problem frequencies take real values. Third, the treatment of the $n=0(m=0)$ mode in tokamaks and A\&A is completely different: whereas this "mode" corresponds to the mean value of the data in A\&A, which has no scientific interest, and actually must be removed from the data so as not to perturb the analysis, in tokamak fusion plasmas such a mode indicates the presence of a major magnetic instability leading to an abrupt vertical displacement of the entire plasma column, which must be correctly detected and prevented, specifically in real-time. Fourth, in the real time applications we consider for JET, a set of data is acquired every $1 \mathrm{~ms}$, therefore the spectral analysis must be completed in an unsupervised manner in the short time between each measurement acquisition.

Evaluating the complex amplitudes $\alpha_{1}$ and the modes number $n_{1}$ of multiple modes in a multi-harmonic spectrum is a very difficult problem, even if the number of modes in the input spectrum is actually known a-priori. The usual way to tackle this problem is performing a best least-square (LS) fitting of the input data. However, this criterion has many local minima for real valued spectral peaks [35-37], 
hence in principle requiring a combinatorial exploration for integer-valued mode numbers $\mathrm{n}_{1}$, and an aposteriori thresholding scheme to differentiate the "correct" from the "wrong" solutions, which is very much CPU-time consuming and cannot possibly be adapted for real-time applications on the submillisecond time scale required for the analysis of the JET measurements. The only possibility to solve this problem is to provide an estimate for the amplitudes of all possible modes numbers in the range $\{-\mathrm{K}, \ldots, \mathrm{K}\}$ (where $|\mathrm{K}|$ is much larger than the maximum mode number that can be conceivably present in the input spectrum), at the same time enforcing that most of these modes actually have a null amplitude, i.e. a utilizing a Sparse Approximation.

Formally, Sparse Representations [12, 38, 39] are representations that account for all information in the input data with a linear combination of a small number of elementary signals called atoms. The atoms set does not form a basis as the number of atoms exceeds the dimension of the signal space, so any signal can be represented by more than one combination of different atoms. Among all these various possible combinations, the one with the smallest number of atoms is the Sparse Representation of the signal. The Sparse Approximations of signals is the version of the Sparse Representations adapted to noisy data. Theoretically, the Sparse Approximation problem consists of minimizing the criterion:

$$
J_{0}(x)=\|\mathbf{y}-W \mathbf{x}\|^{2}+\gamma\|\mathbf{x}\|_{0}
$$

Here $\mathbf{y}=\left[\mathrm{y}_{1}, \mathrm{y}_{2}, \ldots, \mathrm{y}_{\mathrm{P}}\right]^{\mathrm{T}}$ is the vector of data taken at position $\phi_{\mathrm{p}} ; \mathbf{x}=\left[\mathrm{x}_{1}, \mathrm{x}_{2}, \ldots, \mathrm{x}_{\mathrm{M}}\right]^{\mathrm{T}}$ is the vector of complex amplitudes, $\mathbf{W}=\left[\mathbf{w}_{1}, \mathbf{w}_{2}, \ldots, \mathbf{w}_{\mathrm{M}}\right]$ is a matrix where the vector $\mathbf{w}_{\mathrm{k}}$ corresponds to the $\mathrm{k}$-th atom, the L0-norm of $\mathbf{x}:\|\mathbf{x}\|_{0}=\#\left\{k,\left|x_{k}\right| \neq 0\right\}$ is the number of non-zero components of $\mathbf{x}$ and $\gamma$ is a penalization parameter. However, to minimize this criterion, one must sift through all possible combinations of elementary signals, which is intractable for large M. Hence, two kinds of methods have been proposed to get round this problem. The first one, often called greedy pursuit algorithms, iteratively adds atoms to the approximation of the signal to improve this approximation [40]. The second one, often called convex relaxation, replace the L0-norm in criterion (A4a) with another penalization term such that the criterion may be minimized more easily. In our work, we follow this convex relaxation approach, using the classically used L1-norm (see for instance $[12,38,39]$ ) as it leads to the criterion:

$$
J_{0}(x)=\|\mathbf{y}-W \mathbf{x}\|^{2}+\lambda\|\mathbf{x}\|_{1}=\|\mathbf{y}-W \mathbf{x}\|^{2}+\lambda \sum_{k=-K}^{K}\left(\left|x_{k}\right|\right)
$$


It can then be easily shown that the criterion of eq.(A4b) is convex, therefore has no local minima, but, as the number of unknowns may be larger than the number of data, this criterion is not strictly convex, i.e. the solution cannot be a-priori guaranteed to be unique. In practice, minimizing this L1-norm penalized LS fitting criterion is much easier than minimizing the original one based on the L0-norm, and many computationally efficient algorithms have been developed, some of which can be made compatible with a real-time system using a $1 \mathrm{kHz}$ clock-time. However, minimizing eq.(A4b) does not necessarily lead to the same solution than minimizing eq.(A4a). Theoretical conditions guaranteeing the equivalence of both solutions have been established, which are based on properties of the matrix $\mathbf{W}$, so depend on the specificities of the problem being considered.

The choice of the family of atoms is critical in the Sparse Representations of signals as, with an appropriate choice, these atoms might be well adapted to the signal to be analyzed and might lead to a matrix $\mathbf{W}$ with good analytical and numerical properties. For example, it can be shown that if the signal can be represented with $\|\mathbf{x}\|<(1+1 / \mu) / 2$ components, with $\mu=\max _{\mathrm{k} \neq 1}\left(\left|\mathbf{w}_{\mathrm{k}}{ }^{\mathrm{H}} \mathbf{w}_{\mathrm{l}}\right|\right)$, where $\mathbf{W}^{\mathrm{H}}$ is the Hermitian transposition of $\mathbf{W}$, then minimizing eq.(4b) will lead to select the same atoms than the solution minimizing eq.(A4a) [38]. For these reasons, the matrix $\mathbf{W}$ is often chosen as a union of incoherent basis, such as wavelets, Diracs, pure sine waves, or other mathematical approaches.

In our case, the atoms are imposed by the model setup in eq.(A3) to be pure complex exponential waves, $\mathbf{W}=\left\{\exp \left(\mathrm{in}_{\mathrm{k}} \phi_{\mathrm{p}}\right)\right\}_{\mathrm{p}, \mathrm{k}}$, for $\mathrm{p}=\{1, \ldots, \mathrm{P}\}$ and $\mathrm{k}=\{1, \ldots, \mathrm{M}\}$, with $\mathrm{n}_{\mathrm{k}}=\mathrm{k}-\mathrm{K}+1$ and $\mathrm{M}=2 \mathrm{~K}+1$. Due to the irregular sampling, the atoms are strongly correlated. Indeed, it can be shown that $\left|w_{k}^{H} w_{l}\right|=\hat{W}\left(n_{k}-n_{l}\right)$, so that it corresponds to regular samples of the spectral window. As $\hat{W}(v)$ may take values greater than $1 / 3$ (as shown in figA1), the previous condition guarantees exact detection only if the signal consists of a single mode number. Nevertheless, it has been shown from many simulations and analysis of measurements using comparison between different numerical methods that such a solution generally gives very satisfactory results in terms of detection, even in the case of multiple modes (see for instance $[12,25]$ and [31, part-2]. Moreover, for irregular sampling, uniqueness of the global minimizer is almost surely guaranteed if it has less than $\mathrm{P} / 2$ non-zero components, where $\mathrm{P}$ is the data size [12].

In terms of amplitude estimations, it has been shown $[11,12]$ that minimizing eq.(A4b) leads to an under-estimation of the amplitudes of the detected mode numbers due to the L1-norm penalization term of eq.(A4b). Thus, an a-posteriori LS estimation of these amplitudes can be (and usually is for postpulse analysis) performed in a second step within the calculations, after the modes are actually detected. The amplitudes of the detected modes are computed, minimizing the least square criterion 
$\left\|\mathbf{y}-\mathbf{W}_{\text {DET }} \mathbf{x}_{\mathrm{DET}}\right\|^{2}$ where only the non-zero amplitudes of the optimization step are preserved in $\mathbf{x}_{\mathrm{DET}}$. Note that this a-posteriori amplitude estimation step is not an absolute necessity for the real-time analysis, as its main objective is to detect the actual modes, their mode numbers and frequency width, and not to precisely estimate their absolute amplitudes, a scaled value being sufficient for these purpose. A real-time implementation of the proposed modes detection method requires not only an efficient optimization algorithm to minimize eq.(A4b) but also, even more importantly for a frequencydegenerated spectrum, an efficient unsupervised tuning of the penalization parameter $\lambda$. Many numerical algorithms are available to minimize criteria such as those of eq.(A4b) for Sparse Approximations. While for real-valued unknowns $\mathbf{x}_{\mathrm{k}}$ this problem can be written as a classical Quadratic Program, for complex-valued unknowns $\mathbf{x}_{\mathrm{k}}$ it corresponds to a Second-Order Cone Program [39]. An algorithm based on an iterative Block Coordinate Descent procedure has been previously proposed [11, 12], and implemented in the SparSpec code. This procedure consists of performing successive one-dimensional minimization steps with respect to each complex-valued unknown $\mathbf{x}_{\mathrm{k}}$, where each one-dimensional minimization has an explicit solution. This algorithm is very efficient and a correct solution can be typically found in less than $1 \mathrm{~ms}$ using the rather modest computational resources available to process real-time JET data.

The penalization parameter $\lambda$ is related to the noise level and requires an appropriate tuning, since it increases the penalty for those solutions which invoke a large number of modes. It can be shown that (a) for $\lambda>\lambda_{\text {MAX }}=\max \left(\left|W^{H} y\right|\right)=\max _{k}\left(\left|\hat{y}\left(n_{k}\right)\right|\right)$, the minimizer $\mathbf{x}_{\text {MIN }}$ of eq.(A3b) is identically zero, i.e. the unique solution has no detected modes; and (b) for a given $\lambda$, the minimizer $\mathbf{x}_{\mathrm{MIN}}$ of eq.(A4b) satisfies $\max \left(\left|W^{H}\left(y-W x_{\text {MIN }}\right)\right|\right)=\max _{k}\left(\left|\hat{r}\left(n_{k}\right)\right|\right) \leq \lambda$, where $\mathbf{r}=\mathbf{y}-\mathbf{W} \mathbf{x}_{\text {MIN }}$ is called the residual (data minus the model corresponding to the estimated modes). Hence $\lambda$ can be interpreted as the maximum peak amplitude allowed in the periodogram of the residual, and choosing $\lambda$ to be a fraction $\lambda_{\mathrm{NORM}} \in[0$, 1] of the maximum of the periodogram of the data $\lambda=\lambda_{\mathrm{NORM}} \times \max \left(\left|\mathbf{W}^{\mathrm{H}} \mathbf{y}\right|\right)$, ensures the periodogram of the residual $\mathbf{r}$ to be lower up to this fraction relatively to the maximum of the data periodogram. Hence knowledge of the noise level in the measurements helps to determine the optimum value for $\lambda_{\text {NORM }}$ to be used for real-time and post-pulse analysis of MHD fluctuation data. For real-time MHD analysis at JET, the most suitable value for $\lambda_{\text {NORM }}$ was determined to be $\lambda_{\text {NORM }}=0.85$ as this value allows for a very rapid convergence of the optimization algorithm and is sufficient to detect and discriminate multiple modes whose amplitudes are of interest for MHD diagnostic purposes. 


\section{References}

1. Various Authors, "Progress in the ITER Physics Basis", Special Issue of Nucl. Fusion 47 (2007).

2. E.J.Strait, E.D.Fredrickson, J-M.Moret, M.Takechi, Fus. Sci.Tech. 53(2) (2008), 304.

3. J.Wesson, Tokamaks, $3^{\text {rd }}$ edition, (Oxford Science Publication, Oxford, 2003), p.617.

4. A.Fasoli et al., Phys. Rev. Lett. 75 (1995), 645.

5. A.Fasoli, D.Testa et al., Plasma Phys. Control. Fusion 44 (2002), 159.

6. D.Testa and A.Fasoli, Nucl. Fusion 41 (2001), 809.

7. D.Testa et al., Nucl. Fusion 50 (2010), 084010.

8. T.Panis, D.Testa et al., Nucl. Fusion 50 (2010), 084019.

9. P.Lauber, S.Günter et al., Paper THW/P7-08, $23^{\text {rd }}$ IAEA Fusion Energy Conference, Daejeon, Republic of Korea, 11-16 October 2010.

10. D.Testa et al., The new Alfvén Wave Active Excitation System at JET, Proceedings $23^{\text {rd }}$ SOFT Conference 2004 (weblink: http://infoscience.epfl.ch/record/143354/files/).

11. S.Bourguignon, H.Carfantan, T.Böhm, Astronomy and Astrophysics 462 (2007), 379.

12. S.Bourguignon, H.Carfantan, J.Idier, IEEE Journal of Selected Topics in Sig. Proc. 1 (2007), 4.

13. F.Marvasti, Non-Uniform Sampling: Theory and Practice, Springer-Verlag (2001).

14. N.R.Lomb, Astrophysics and Space Science 39 (1976), 447.

15. J.D.Scargle, The Astroph. Journal 263 (1982), 835.

16. D.Roberts, J.Lehar and J.Dreher, The Astron. Journal 93 (1987), 968.

17. G.Foster, The Astron. Journal 109 (1995), 1889.

18. J.S.Kim et al., Plasma Phys. Control. Fusion 41 (1999), 1399.

19. M.Hole and L.Appel, Plasma Phys. Control. Fusion 49 (2007), 1971.

20. F.M.Poli et al., Plasma Phys. Control. Fusion 50 (2008), 095009.

21. E.P.Wigner, Phys. Rev. 40 (1932), 749.

22. H.Choi, W.Williams, IEEE Trans. Acoust. Speech Signal Process 37 (1989), 86.

23. N.E.Huang et al., Proc. Royal Society London A454 (1998), 903.

24. S.Zegenhagen, A. Werner et al., Plasma Phys. Control. Fusion 48 (2006), 1333.

25. A.Klein, H.Carfantan, D.Testa, et al., Plasma Phys. Control. Fusion 50 (2008), 125005.

26. D.Testa, H.Carfantan, A.Goodyear, et al., EuroPhysics Letters 92 (2010), 50001.

27. S.Sharapov, D.Testa et al., Phys. Lett. A 289 (2001), 127.

28. W.W.Heidbrink, Phys. Plasmas 9(5) (2002), 2113.

29. G.Vlad, S.Briguglio, G.Fogaccia, F.Zonca, Nucl. Fusion 46 (2006), 1. 
30. A.Fasoli et al., Nucl. Fusion 36(2) (1996), 258-263.

31. D.Testa et al., Fus. Sci. Tech. 57(3) (2010), 208-237 and Fus. Sci. Tech. 57(3) (2010), 238-273.

32. J.Lafler and T.D.Kinman, The Astrophys Journal Suppl. 11 (1965), 216.

33. M.M.Dworetsky, Mon. Not. R. Astron. Soc. 203 (1983), 917.

34. P.Stoica, R.L.Moses, B.Friedlander, and T.Söderström, IEEE Trans. ASSP 37 (1989), 378.

35. J.-J.Fuchs, IEEE Trans. Inf. Theory 51 (2005), 3601.

36. D.L.Donoho, M.Elad, and V.N.Temlyakov, IEEE Trans. Inf. Theory 52 (2006), 6.

37. J.A.Tropp, IEEE Trans. Inf. Theory 52 (2006), 1030.

38. D.Donoho, Journal of Communications on Pure \& Applied Math. 59 (2006), 797.

39. D.M.Malioutov, A sparse signal reconstruction perspective for source localization with sensor arrays, Master Thesis, MIT, July 2003.

40. J.A.Tropp, IEEE Trans. Inf. Theory 50 (2004), 2231-2242. 


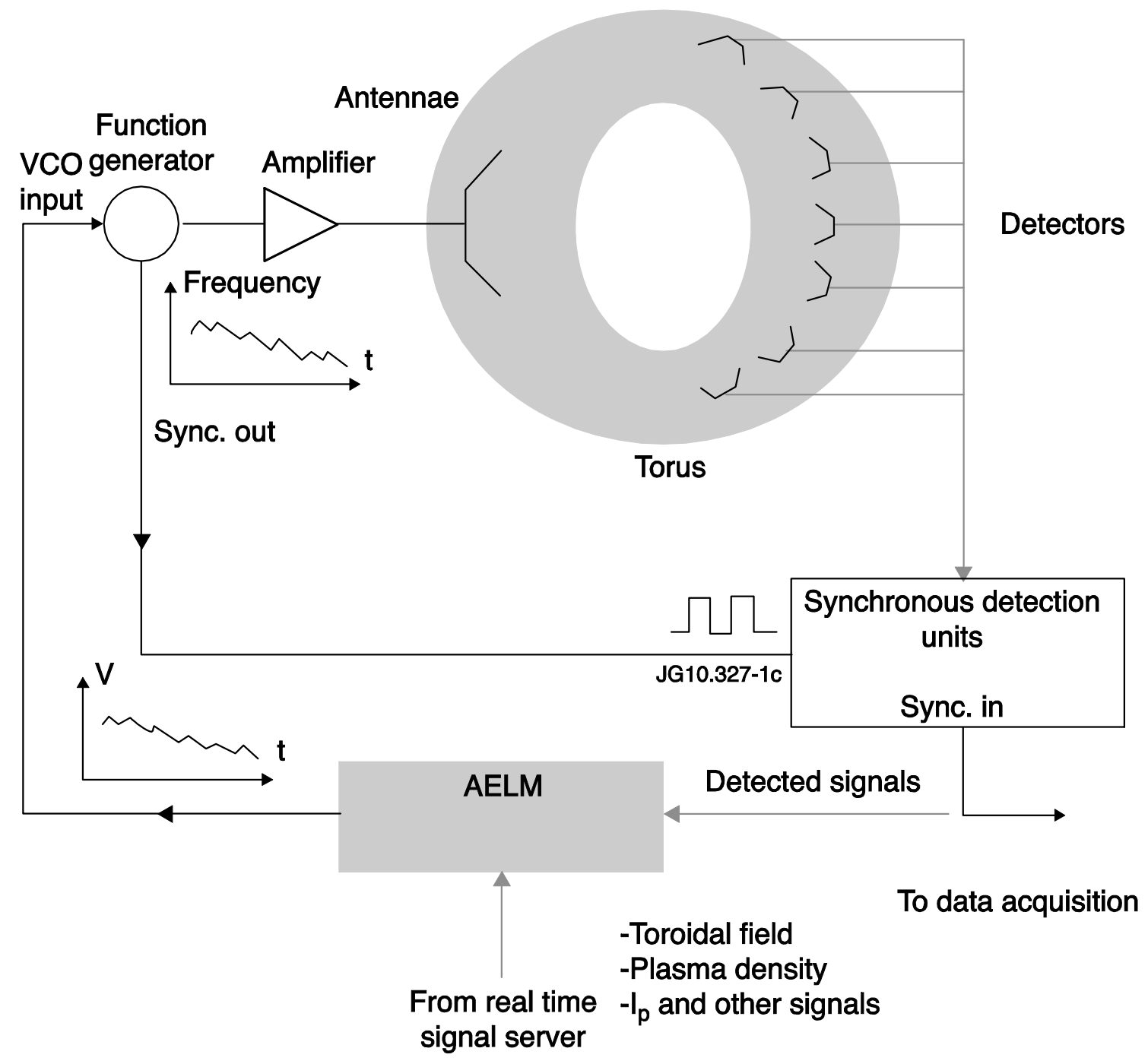

Figure1. A schematic overview of the AEAD system in JET. The toroidal field, plasma density and plasma current $\left(\mathrm{I}_{\mathrm{p}}\right)$ are required to compute in real-time an initial guess for the frequency of Alfvén Eigenmodes. This value is then converted as a time-dependent voltage $V(t)$ and is sent as a Voltage Controlled Oscillator (VCO) input signal to the function generator which, in turn, converts it back into a time-dependent AC frequency signal, Frequency $(\mathrm{t})$. This signal then drives a high-power amplifier, connected to a set of up to eight in-vessel antennae, and the synchronous detection units via an optical transducer, so that only the portion of the plasma response which has the same frequency of the antenna drive (i.e. it is synchronous with this drive) is detected in real-time, thus avoiding the need for real-time computationally expensive FFT algorithms. 


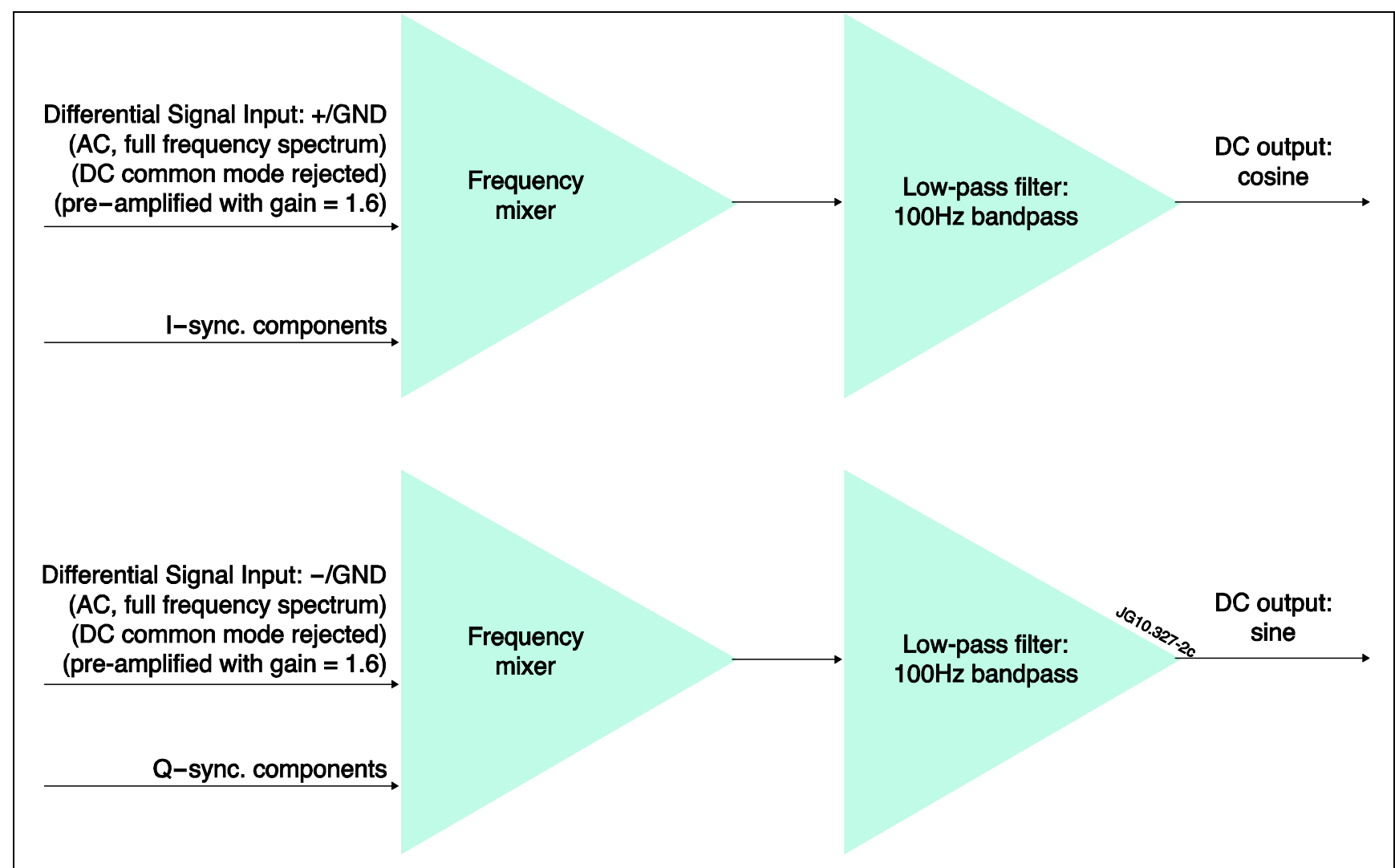

Figure2. A schematic diagram illustrating the AEAD synchronous detection hardware. This hardware is conceptually based upon first removing any DC common mode from the input AC differential signal (with a full frequency spectrum), which is then amplified, and finally applying a low-pass filter to extract only the component in the input signal which is at the desired (i.e. synchronous) frequency.

D.Testa et al., Figure2, PaperAELMforFED2011-V3 


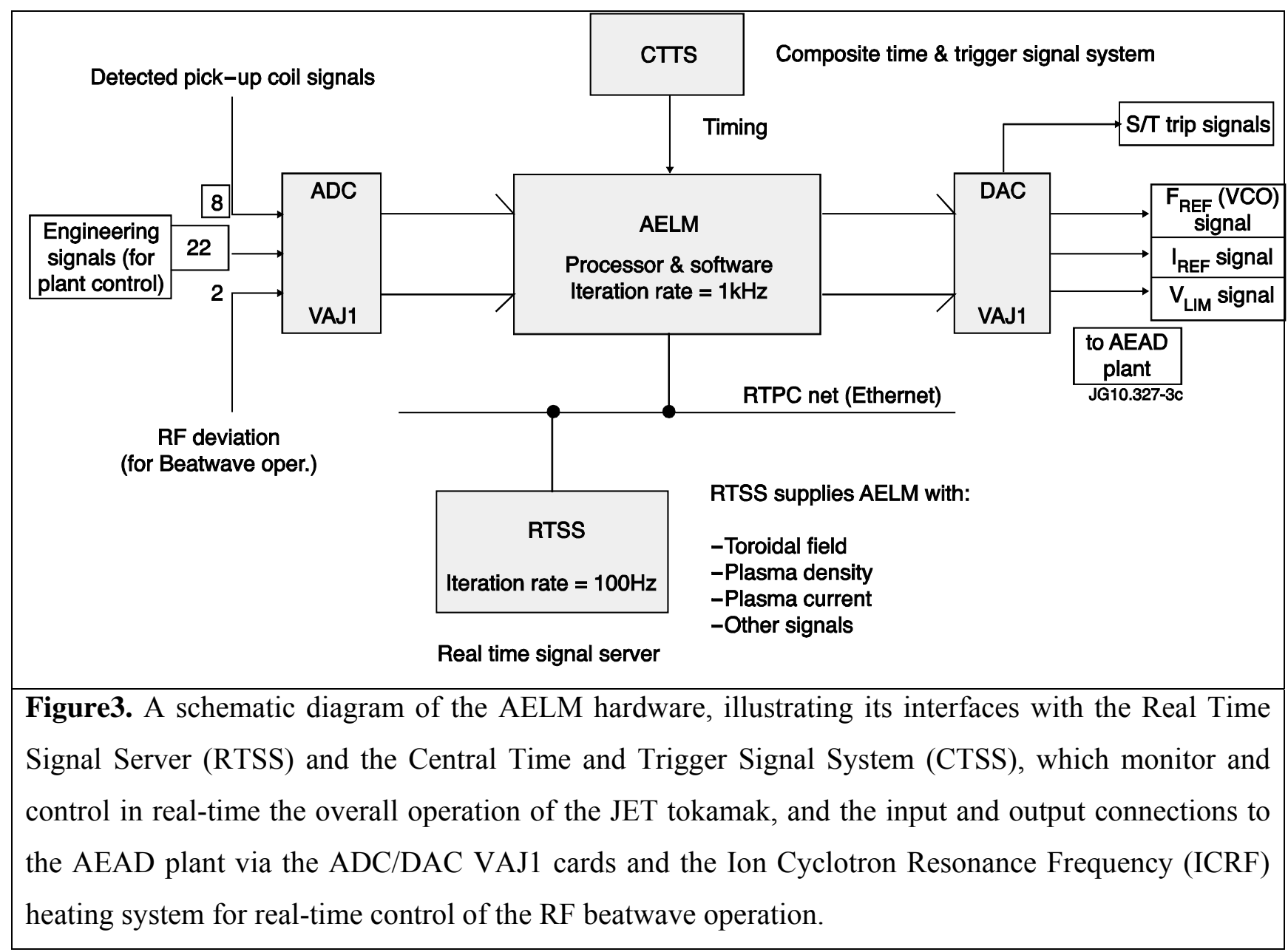

D.Testa et al., Figure3, PaperAELMforFED2011-V3 


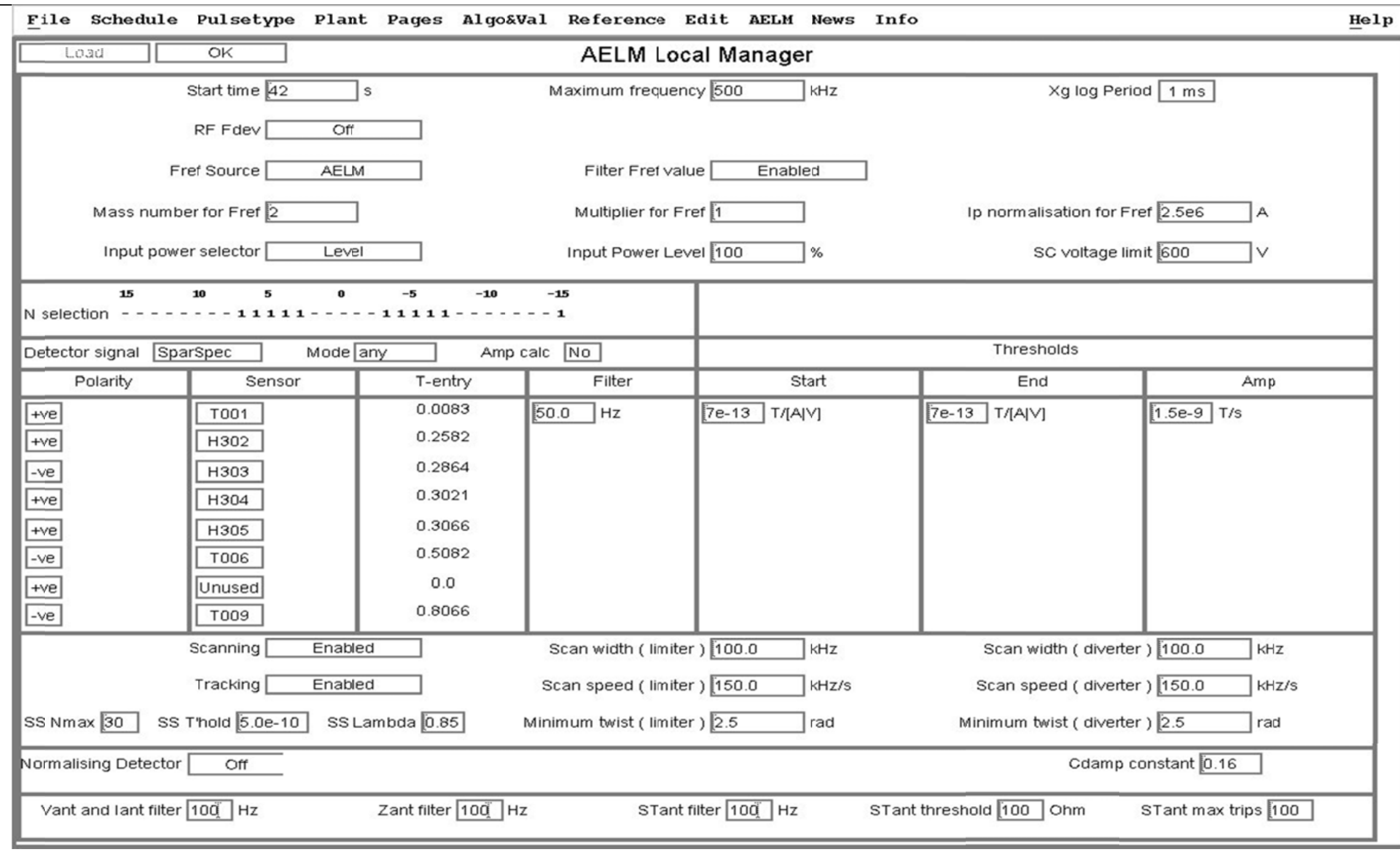

Figure4. The AELM configuration panel, shown here for the SparSpec algorithm and frequency control via the real-time TAE frequency.

D.Testa et al., Figure4, PaperAELMforFED2011-V3 


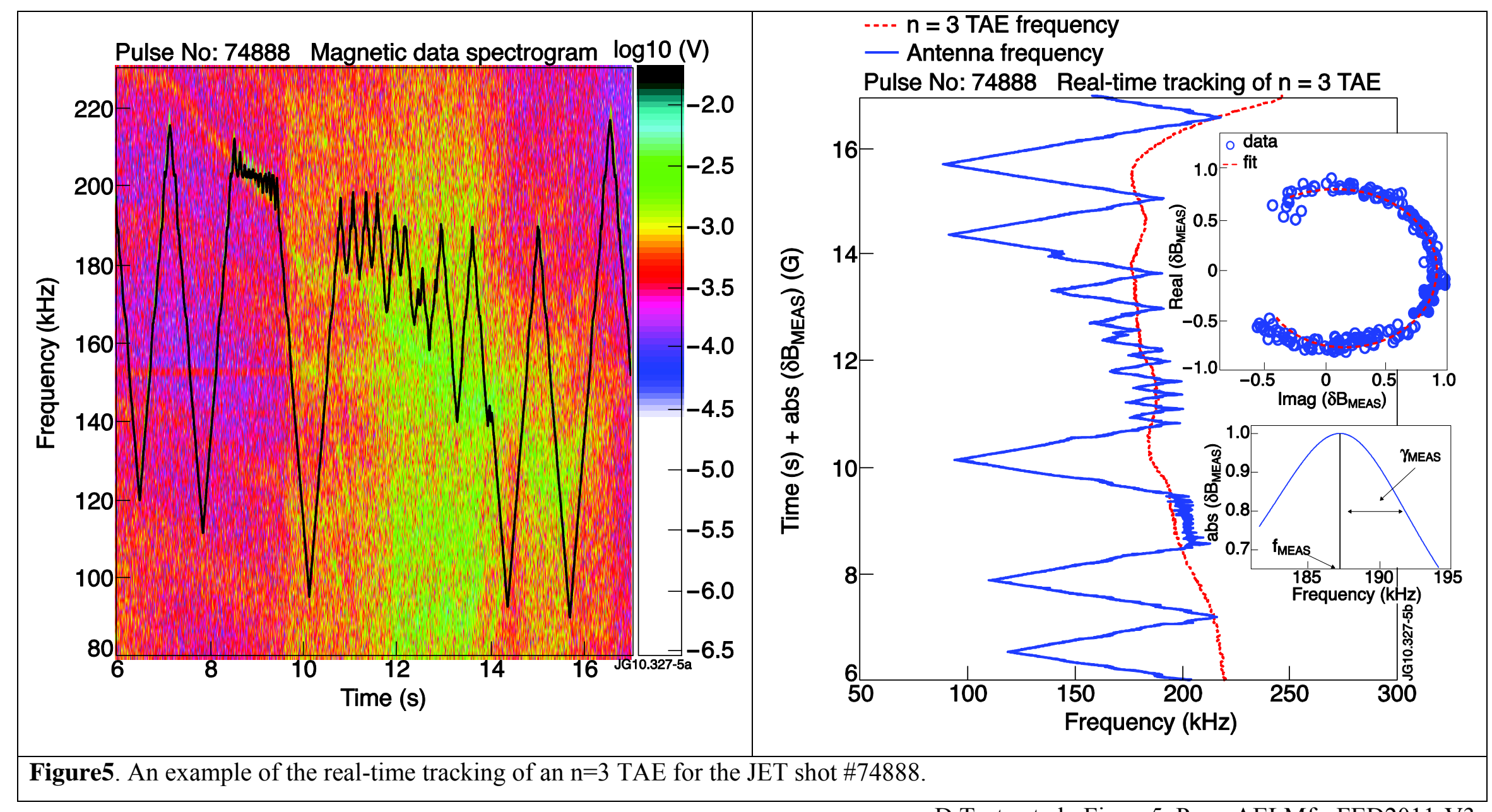

D.Testa et al., Figure5, PaperAELMforFED2011-V3 

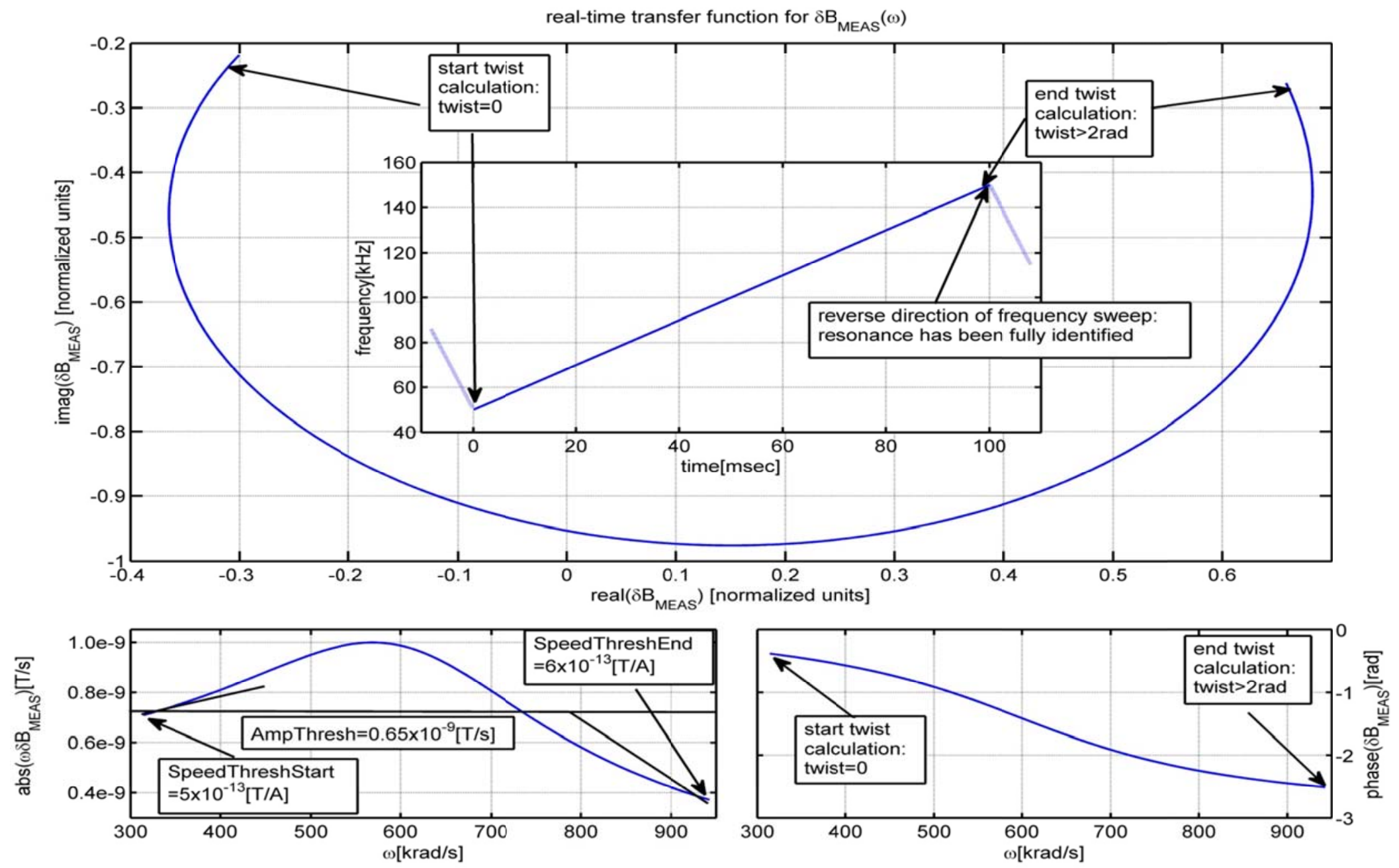

Figure6. A schematic diagram to illustrate the basic ideas of the real-time tracking algorithm.

D.Testa et al., Figure6, PaperAELMforFED2011-V3 


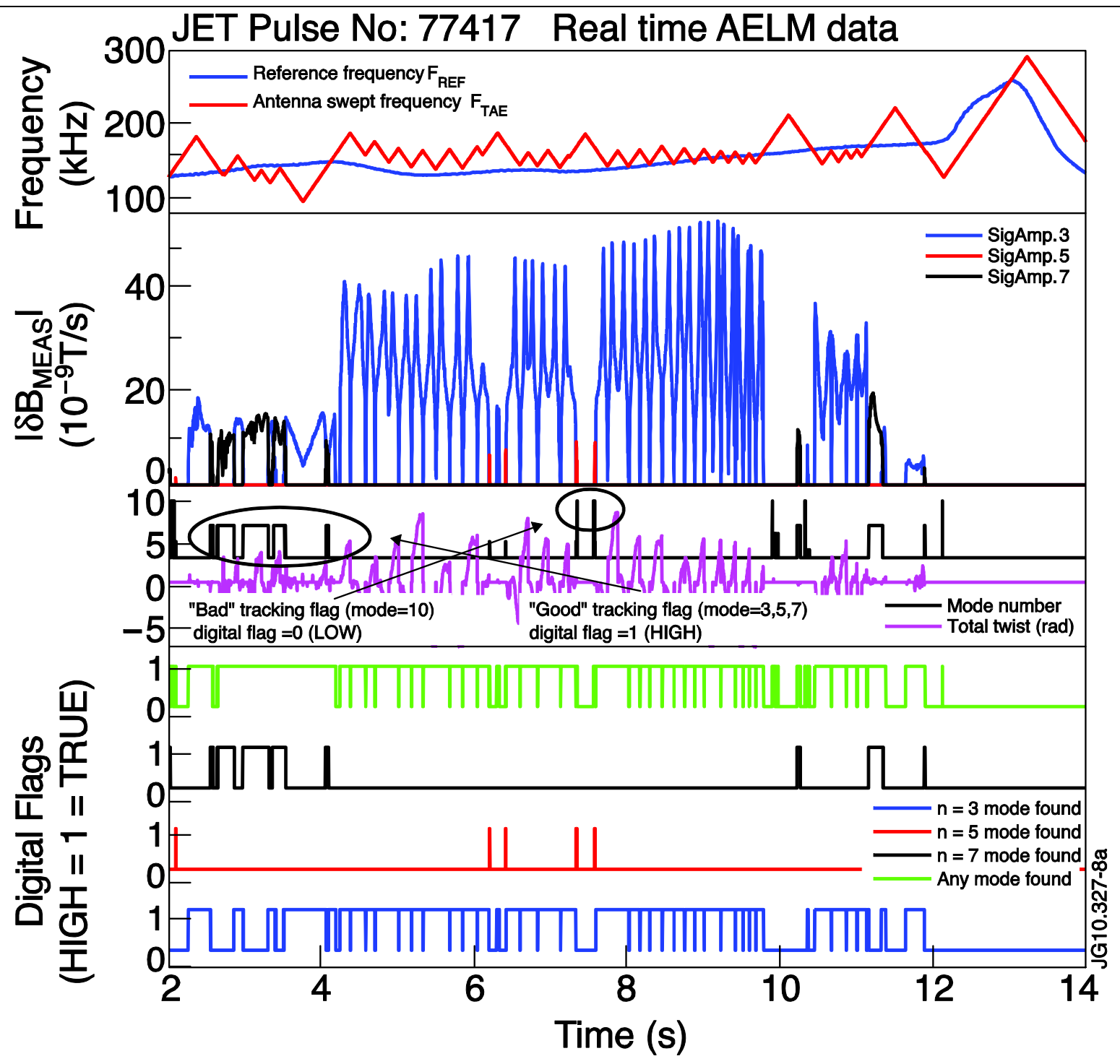

Figure7a. An example of real-time tracking of the individual $n=[3,5,7]$ TAEs for the JET shot \#77417; the signal SigAmp.N shows the amplitude of the $n=N$ modes as detected in real-time; the $n=10$ mode corresponds to bad tracking because its digital validity flag is false $(=0)$; for illustrative purposes, in the bottom frame the green line (label: "any mode found") indicates that a mode has been found, whichever its toroidal mode number may be, hence this flag goes high $(=1)$ concurrently with the appearance of any of the $n=3, n=5$ and $n=7$ modes shown in this figure, and goes low $(=0)$ only when no mode at all has been found with toroidal mode number in the range selected in real-time.

D.Testa et al., Figure7a, PaperAELMforFED2011-V3 


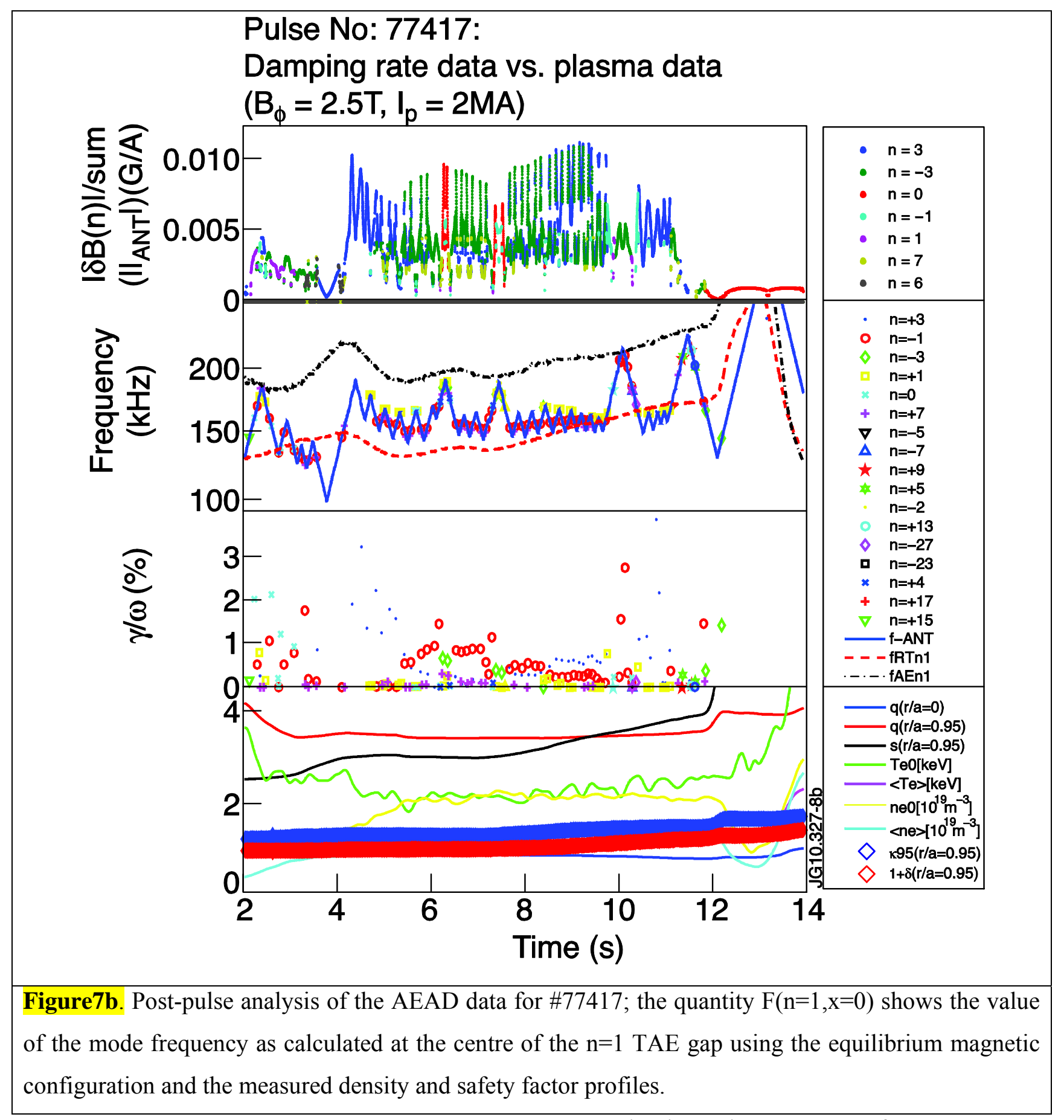

D.Testa et al., Figure7b, PaperAELMforFED2011-V3 

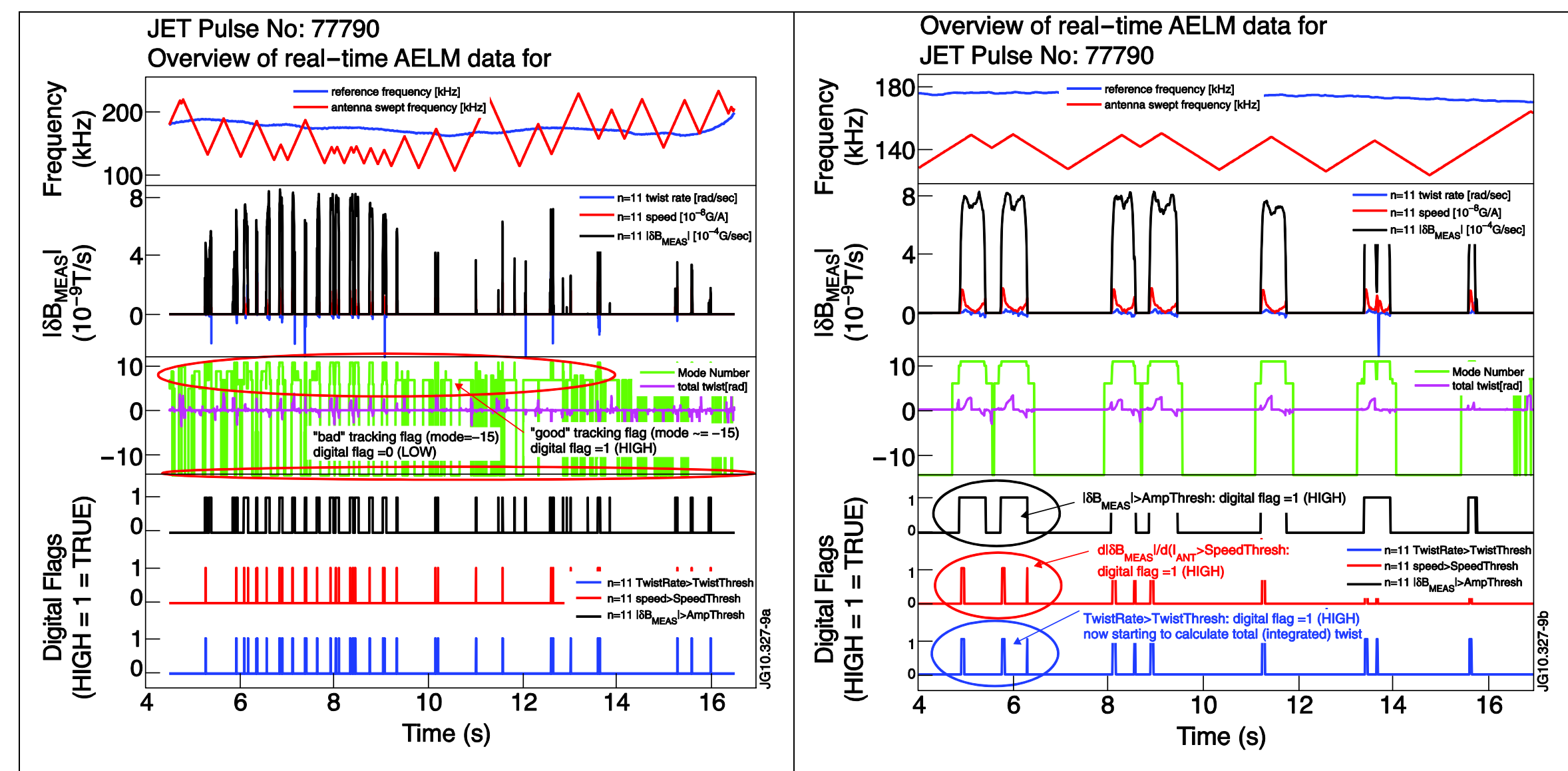

Figure8(a,b). A real-time example of the twist and speed calculations for the JET shot \#77790 and the n=11 TAE mode (fig8a, left frame); the zoom shown in fig8b (right frame) demonstrates that the tracking of the $n=11$ mode only occurs when all the resonance conditions are above the set thresholds, i.e. the digital flags for the mode amplitude and speed are concurrently high (see the bottom frame).

D.Testa et al., Figure8(a,b), PaperAELMforFED2011-V3 


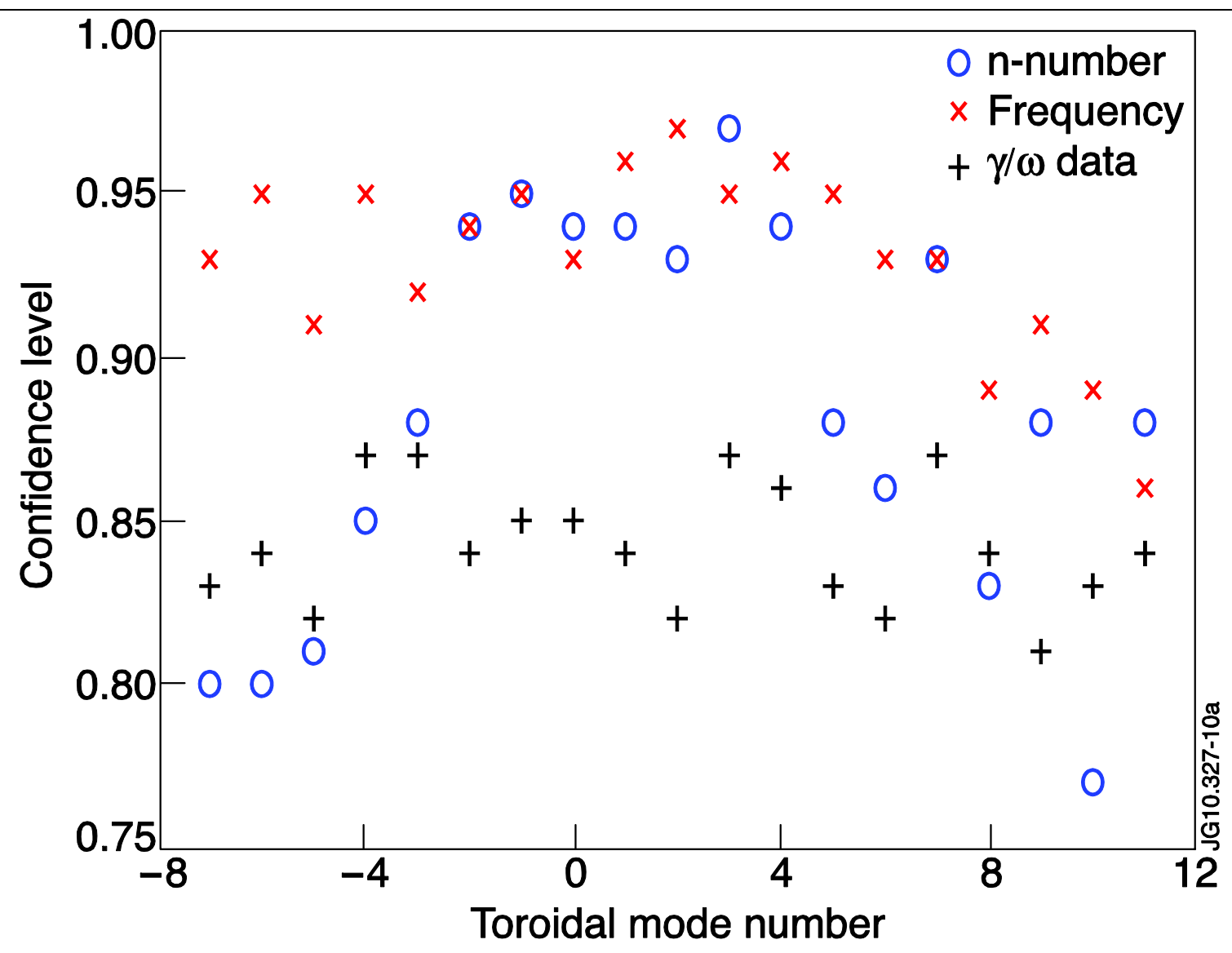

Figure9. Confidence level for the real-time evaluation of the mode number, frequency and damping rate when using the SparSpec tracking algorithm.

D.Testa et al., Figure9, PaperAELMforFED2011-V3 

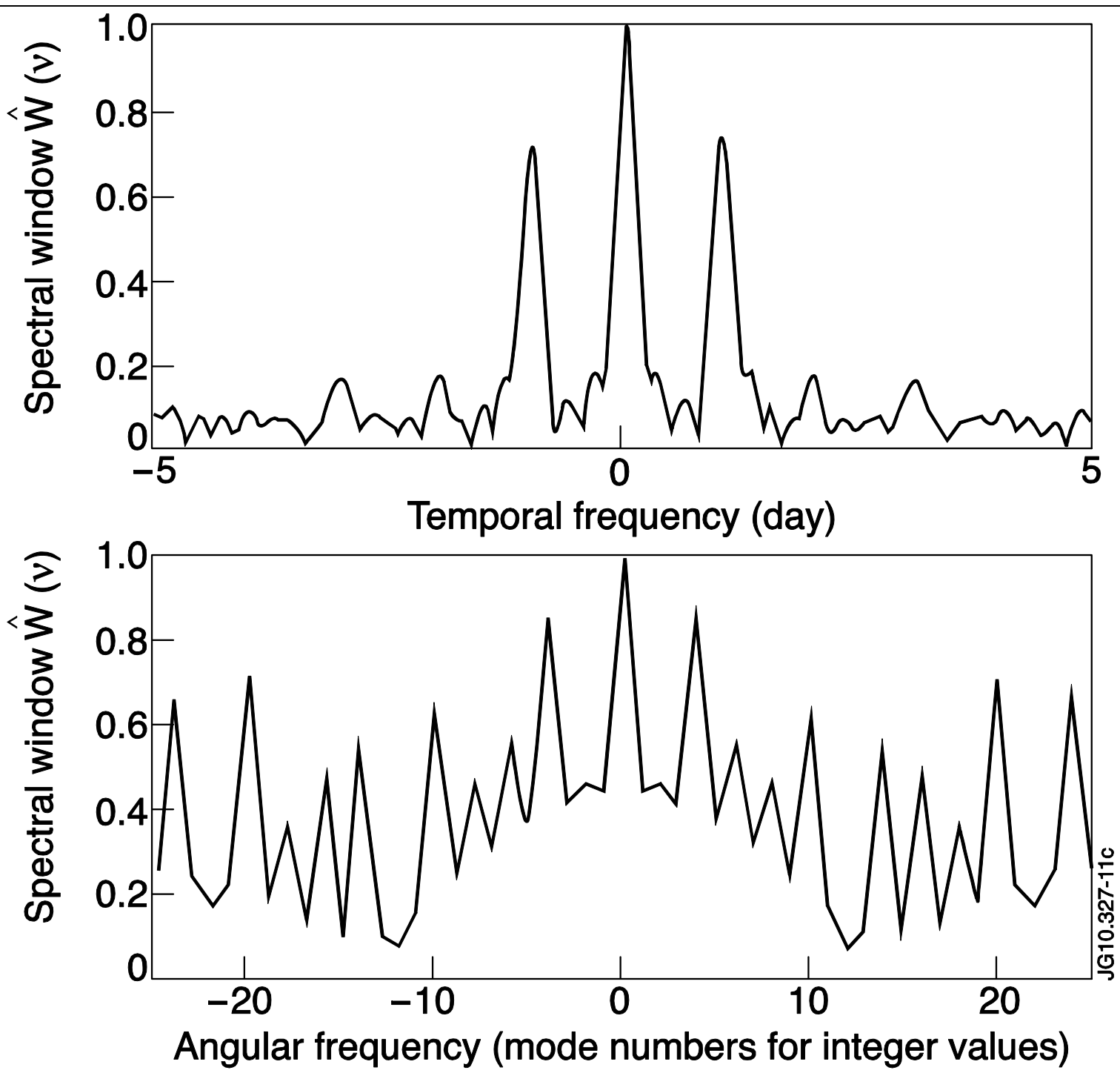

FigureA1. The spectral windows corresponding to an example of astronomical observations during five nights (top) and to the actual positions of the seven magnetic sensors that are sufficiently reliable to be used in the real-time data processing algorithm for the analysis of MHD instabilities (bottom) in JET.

D.Testa et al., FigureA1, PaperAELMforFED2011-V3 